\title{
Design and Simulation of Voltage Source Inverter
}

\author{
Ma Ning*, Wang Niansheng, Liu Weijian, Lai Xiangdong, Liu Yanbin, Xiong Jue \\ Wuhan Electronic Information Institute, Wuhan, China. \\ * Corresponding author. Email: 156449871@qq.com \\ Manuscript submitted March 1, 2019; accepted May 29, 2019. \\ doi: 10.17706/ijcee.2019.11.4.175-179
}

\begin{abstract}
This paper describes the design of the monopole full-bridge voltage source inverter and demonstrates the working principle with the PSPICE simulation waveform, then proposes a more practical optimization. The simulation result ensures that the inverter has less distortion and smaller phase shift, which is adjustable. And it is useful to the real design and circuit optimization.
\end{abstract}

Key words: PSPICE, voltage source inverter, phase shift network.

\section{Introduction}

Due to the exhaustion of the traditional energy, solar energy system (photovoltaic power generation), as a clean and Renewable energy, is a tendency in recent years. And grid connection is the best way to make full use of this energy. The simulation circuit of photovoltaic grid connected power generation with DC/AC convertor and the inverter of the full bridge structure use the monopole frequency multiplication and the SPWM method, which is well known for the less voltage distortion and higher efficiency.

As the Fig. 1 showed, the MCU, PPL and frequency multiplication circuit, primary control circuit, power supply main circuit and feedback circuit are included in the system. In this paper, we use the PSPICE simulation to intuitively illustrate the working principle of the system and optimization in the inverter of master control circuit and power supply main circuit.

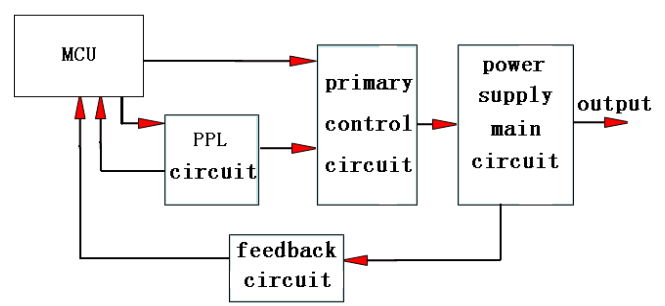

Fig. 1. System chart.

We choose the minimal system board of C8051F020 as the MCU, which would help hardware circuit to implement the function of frequency follow, phase track, over current/undervoltage protection, and automatic recovery.

\section{The Simulation of Primary Control Circuit}

As the Fig. 2 showed, the master control circuit can be divided into 3 parts. Part a is the $50 \mathrm{~Hz}$ sine wave which is the output of the MCU's D/A port. Its DC offset is $1.4 \mathrm{~V}$, and the peak value is $1.35 \mathrm{~V}$. This sine wave 
is AC coupled to voltage follower which formed by Integrated Operational Amplifier TLC2272. Hence we get AC sine wave with $0 \mathrm{DC}$ offset and $1.35 \mathrm{~V}$ in the peak value.

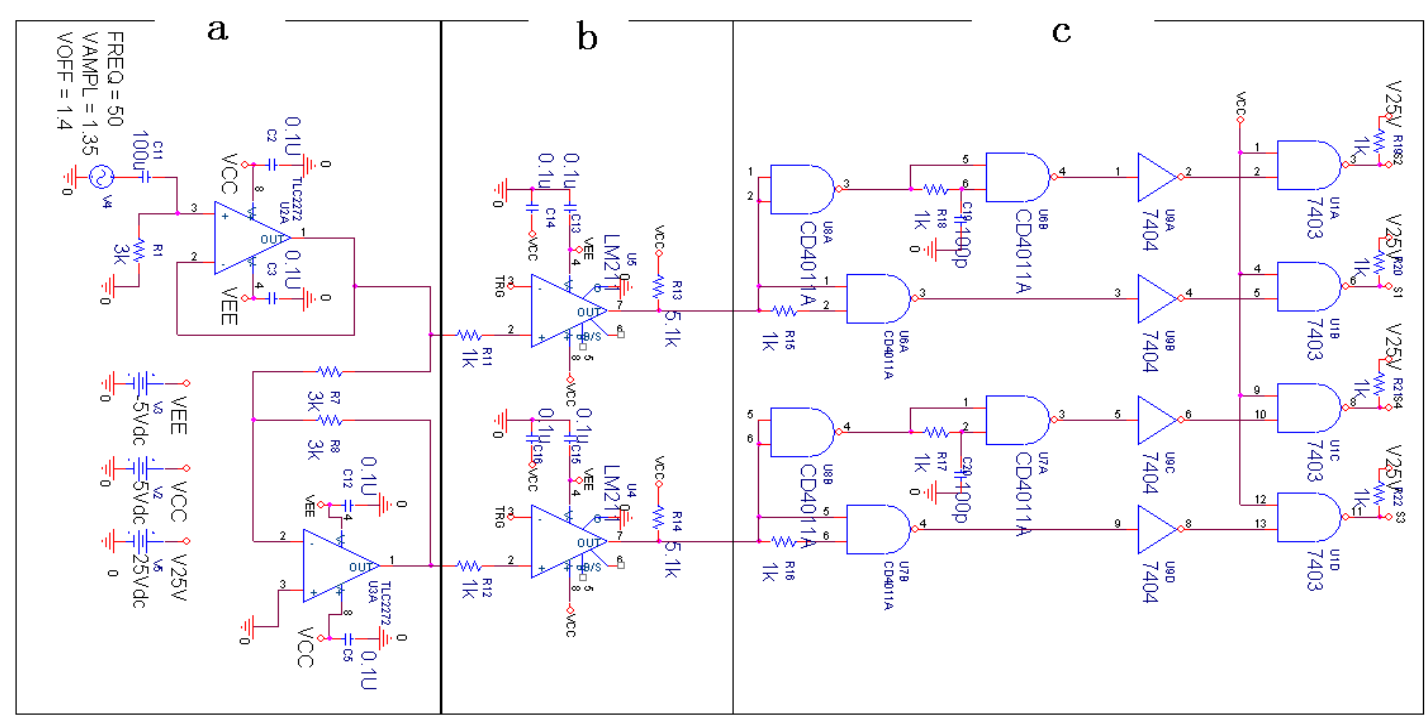

Fig. 2. The master control circuit.

And then put this signal into an input pin of the comparator in Part b. Another input pin of the comparator is showed by the Fig. 3. The positive and negative integral circuit produces the positive and negative triangle wave with $1.6 \mathrm{~V}$ peak value and $6.4 \mathrm{KHz}$ frequency, which is showed by Fig. 4. Compare the sine wave with triangle wave, we can get a PWM pulse signal, which showed by Fig. 5 . While part C presents the pulse signal of positive and negative polarity output by the phase logic and the output port is pulled up by OC gate 7403 to $25 \mathrm{~V}$ to make the pulse signal capable to drive MOS FET directly.

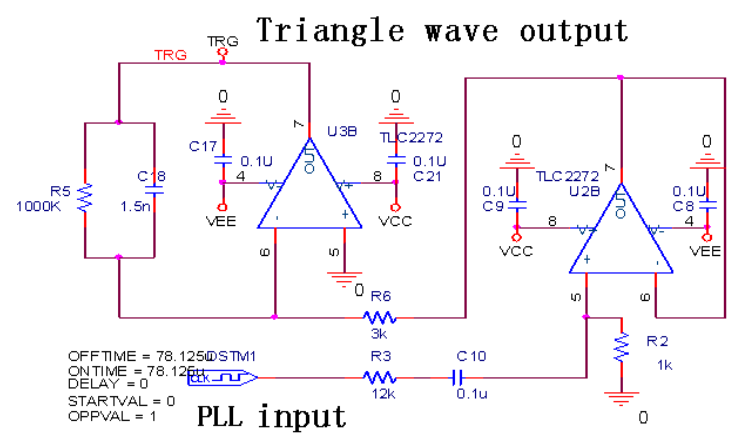

Fig. 3. Triangular waveform generator.

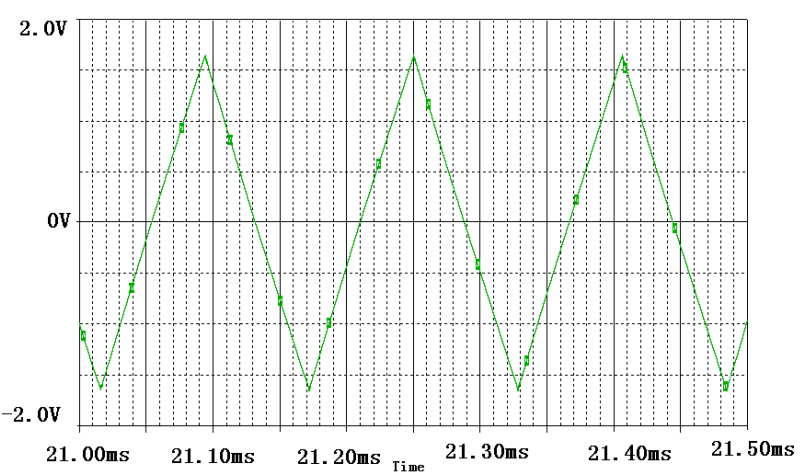

Fig. 4. Triangle wave. 
The input signal of integral circuit in Fig. 3 is generated by MCU and then is phase locked and frequency multiplied by 125 by PPL. The signal is a square wave of $6.4 \mathrm{kHz}$ phase locked. That square wave is captured by follower then integrated to get the positive and negative triangle wave.

\section{The Simulation of Power Supply Main Circuit}

The OC gate in part C of master control circuit is connected by a common pin from MCU. The power supply would stop if the output of the MCU is low and the pulse signal will be always 0 . But if the output of MCU is high and the pulse signal will be normal operation mode. The control pulse showed in part $\mathrm{C}$ is connected to the power main circuit as showed in Fig. 6, which is via the S1-S4 port showed in Fig. 2. And then it is connected with the gate of FET in power supply to control the switching state of FET [1]. The power supply main circuit uses DC power supply and high-power resistance to simulate photovoltaic battery. FET uses IRF350 high-power power supply switching transistor with shorter switching time, high current which is suitable for power supply. The FET gate controlled by the controlling pulse works in switching state and outputs pulse correspondingly. The positive and negative polarity pulse signal showed in Fig. 7 contains $50 \mathrm{~Hz}$ and $6.4 \mathrm{kHz}$ frequency components while the pulse-width is influenced by the modulation of gate [2].

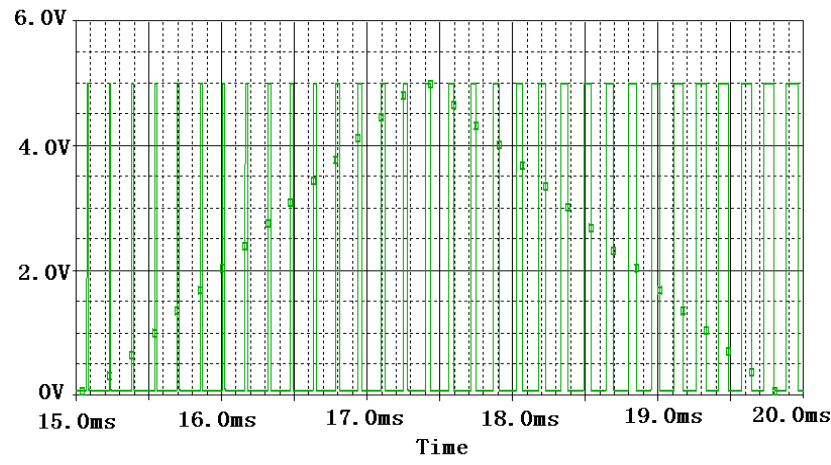

Fig. 5. Drive pulse signal.

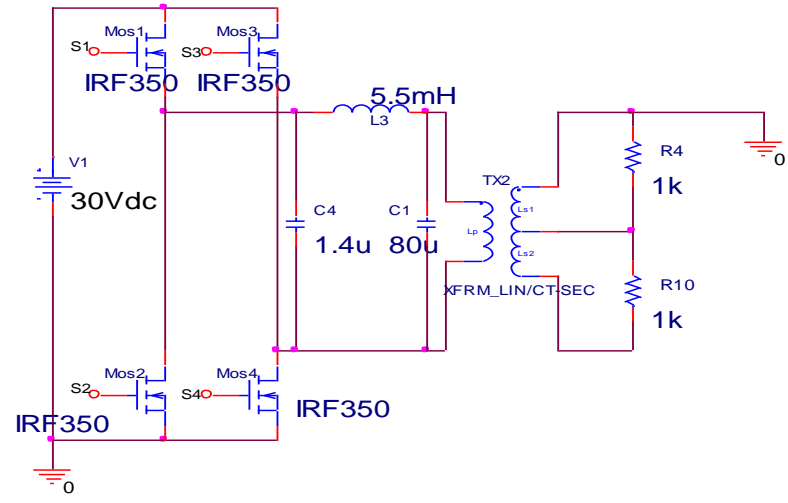

Fig. 6. Power main circuit.

Feed the pulse voltage into the low-pass filter network consists of the capacitors $\mathrm{C} 1=80 \mathrm{uF}, \mathrm{C} 4=1.4 \mathrm{uF}$ and inductor L3=5.5mH showed in Fig. 6 [3]. And if we couple it with transformer we could get the $50 \mathrm{~Hz}$ sine wave showed in Fig. 8. In Fig. 8 the lower wave is the $50 \mathrm{~Hz}$ sine wave transformed by the inverter, and the upper one is the reference signal inputted by the MCU. Comparing them we can get the conclusion that the sine wave outputted by inverter has a little bit distortion and about 7 degree phase lead. But we can adjust it by setting high $\mathrm{Q}$ low-pass filter network and optimizing the value of $\mathrm{C} 1, \mathrm{C} 4$, L3 to reduce the distortion. 


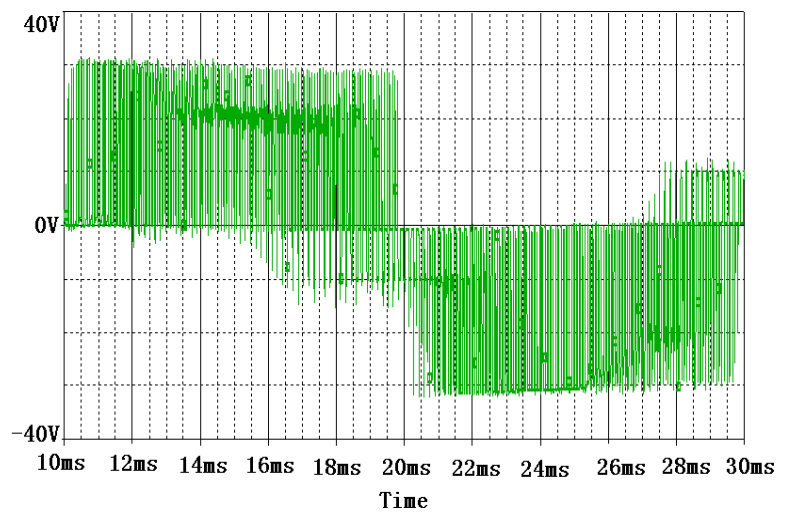

Fig. 7. The positive and negative polarity pulse signal.

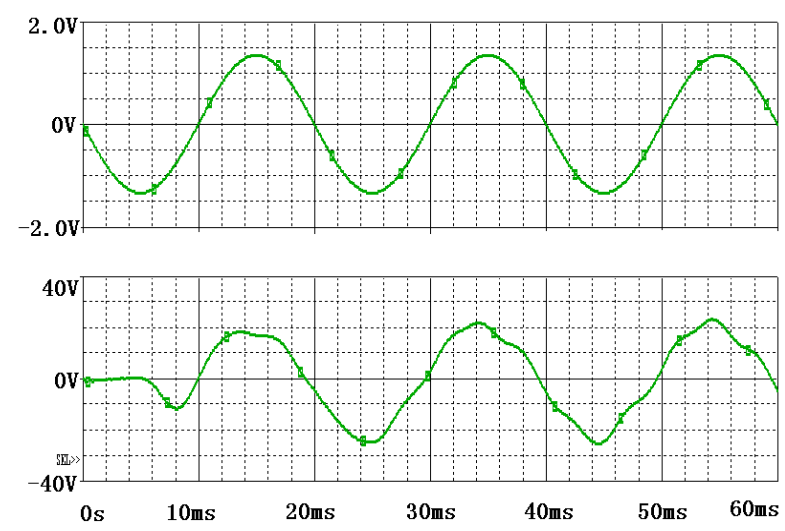

Fig. $8.50 \mathrm{~Hz}$ sine wave of output and input.

\section{The Improvement of Phase Shifting Circuit}

Phase shifting is the key point to the success of the inverter. We can decrease distortion by adding phase shift network. Phase shift network showed in Fig. 9, is consist of 3 parts. The first part is a voltage follower formed by LM324 Integrated Operational Amplifier [4]. The second part is the basic phase shift network form by R3 and C6. The third part is another follower. The output waveform of phase shift network is showed in Fig. 10: the input signal is the green sine wave with a frequency of $50 \mathrm{~Hz}$, and that yellow sine waveform is the wave form output by the phase shift network. As it is shown in the figure, the yellow waveform is lagging behind the green waveform. The lag angle is decided by resistor R3 and capacitor C6. If you want to use the network, the R3 should be a precision adjustable resistor. So that we can adjust the phase difference between the power output voltage and the reference AC, as auxiliary phase track [5].

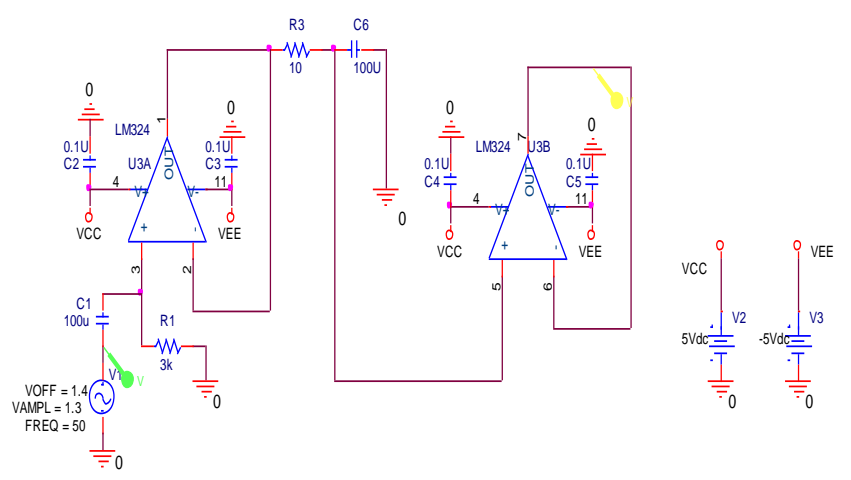


Fig. 9. Phase shift meshwork.

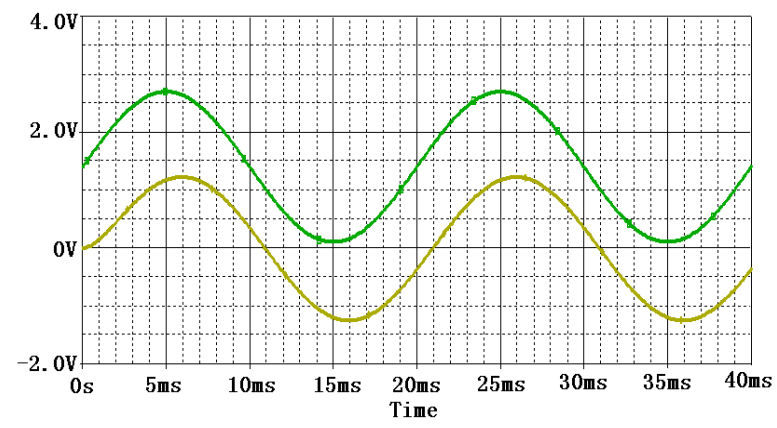

Fig. 10. Output waveform of phase shift network.

\section{Conclusion}

In this paper, we use PSPICE to simulate the working principle and waveform of the master control circuit of inverter and power supple circuit. All of the circuit simulation in this paper comes from the design of an actual circuit. The simulation results guide the actual circuit successfully. Due to limited space, the details about the feedback circuit, the PPL frequency multiplication and the control program of single chip microcomputer are skipped.

\section{References}

[1] Shen, Y., Xu, W., \& Zhao, W. (2004). Study of control rules of single input single phrase SPWM modulation photovoltaic synchronization power generation system. Solar Energy Transaction, 25(6), 794-797.

[2] Song, Q., Liu, W., \& Yang, A. (2003). The design of the LC filter from high-capacity PWM voltage source inverter. Qinghua University Transaction (science), 43(3), 345-348.

[3] Wang, X., Ruan, X., \& Liu, S. (2011). Control strategy for grid-connected inverter to suppress current distortion effected by background harmonics in grid voltage. Proceedings of the CSEE: Vol. 6.

[4] Zhang, G., Tang, X., Zhou, L., \& Qi, Z. (2011). Research on complementary PWM controlled buck/boost bi-directional converter in supercapacitor energy storage. Proceedings of the CSEE: Vol. 6.

[5] Torabzad, S., Babaei, E., \& Kalantari, M. (2010). Z-source inverter based dynamic voltage restorer. Proceedings of the 1st Power Electronic \& Drive Systems \& Technologies Conference.

Ma Ning was born on 18th July, 1975 in Wuhan, Hubei. He holds the master degree of electrical engineering \& electronics and is an associate professor in power electronics of Wuhan Electronic Information Institute, Wuhan, China. Now the author's memberships are engaged in scientific research projects of power supply development. 